\title{
Associação de diferentes abordagens da laserterapia de baixa potência no tratamento da deiscência cirúrgica de artroplastia do joelho
}

\author{
Association of different approaches to low level laser therapy in the treatment of surgical \\ dehiscence of knee arthroplasty \\ Asociación de diferentes enfoques a la laserterapia de baja potencia en el tratamiento de la \\ deshiscencia quirúrgica de la artroplastia de rodilla
}

Recebido: 23/08/2021 | Revisado: 02/09/2021 | Aceito: 06/09/2021 | Publicado: 07/09/2021

Karina Alexandra Batista da Silva Freitas
ORCID: https://orcid.org/0000-0002-3413-8468
E-mail: k.freitas@unesp.br
Eliana Maria Minicucci
Hospital das Clínicas da Faculdade de Medicina de Botucatu, Brasil
ORCID: https://orcid.org/0000-0002-4664-6929
E-mail: eminicucci@ gmail.com
Vanderlei Salvador Bragnato
Hospital das Clínicas da Faculdade de Medina de Botucatu, Brasil
ORCID: https://orcid.org/0000-0003-4833-239X
Universidade de São Paulo, Brasil
E-mail: vander@ @ifsc.usp.br
Rosane de Fátima Zanirato Lizarelli
ORCID: https://orcid.org/0000-0003-0418-8381
Núcleo Integrado de Laser em Odontologia, Brasil
E-mail: lizarelli@ @otmail.com

\begin{abstract}
Resumo
O objetivo do trabalho foi demonstrar a associação da fotobiomodulação, terapia fotodinâmica, terapia a laser a vácuo, irradiação de sangue com laser transcutâneo e curativo gel de Polihexametileno Biguanida tópicos no tratamento de deiscências de artroplastia de joelho. A metodologia utilizada foi um relato de caso descritivo, com coleta de dados do tratamento da paciente por meio do prontuário eletrônico. Foram analisados os sinais e sintomas, apresentados pela paciente, bem como as características da ferida. Dessa forma, os protocolos utilizados foram prescritos de forma individualizada, garantindo um atendimento diferenciado para a paciente. Foi autorizado pelo comitê de ética e a paciente assinou o termo de consentimento livre e esclarecido. Os resultados demostraram que a associação de diferentes abordagens da fotobiomodulação foram eficazes na cicatrização completa da ferida. Após 8 dias, observamos uma diminuição da profundidade do leito, do eritema e da inflamação do tecido ao redor com delimitação das bordas da ferida; bem como o clareamento da pele. Por tratar-se de uma ferida crônica, infectada a cicatrização completa aconteceu em sessenta e três dias. Conclui-se que que a combinação da ação fotodinâmica com pressão negativa estimulando a vascularização e a oxigenação bem como a melhora das condições corporais com SVPBM, promoveu uma recuperação muito mais rápida do que cada ação individual.
\end{abstract}

Palavras-chave: Fotobiomodulação; Deiscência cirúrgica; Terapia fotodinâmica.

\begin{abstract}
The objective of this study was to demonstrate the association of photobiomodulation, photodynamic therapy, vacuum laser therapy, blood irradiation with transcutaneous laser and topical Polyhexamethylene Biguanide gel dressing in the treatment of dehiscence in knee arthroplasty. The methodology used was a descriptive case report, with data collection on the patient's treatment through the electronic medical record. The signs and symptoms presented by the patient were analyzed, as well as the characteristics of the wound. Thus, the protocols used were individually prescribed, ensuring differentiated care for the patient. It was authorized by the ethics committee and the patient signed an informed consent form. The results showed that the association of different photobiomodulation approaches were effective in complete wound healing. After 8 days, we observed a decrease in bed depth, erythema and inflammation of the surrounding tissue, with delimitation of the wound edges; as well as skin whitening. As it was a chronic, infected wound, complete healing took place in sixty-three days. It is concluded that the combination of photodynamic action with negative pressure stimulating vascularization and oxygenation, as well as improving body conditions with SVPBM, promoted a much faster recovery than each individual action.
\end{abstract}

Keywords: Photobiomodulation; Surgical dehiscence; Photodynamic therapy. 


\begin{abstract}
Resumen
El objetivo de este estudio fue demostrar la asociación de la fotobiomodulación, la terapia fotodinámica, la terapia con láser de vacío, la irradiación de sangre con láser transcutáneo y el apósito de gel de polihexametileno biguanida tópico en el tratamiento de la dehiscencia en la artroplastia de rodilla. La metodología utilizada fue un reporte de caso descriptivo, con recolección de datos sobre el tratamiento del paciente a través de la historia clínica electrónica. Se analizaron los signos y síntomas que presenta el paciente, así como las características de la herida. Así, los protocolos utilizados fueron prescritos individualmente, asegurando una atención diferenciada para el paciente. Fue autorizado por el comité de ética y el paciente firmó un consentimiento informado. Los resultados mostraron que la asociación de diferentes enfoques de fotobiomodulación fue efectiva en la cicatrización completa de heridas. A los 8 días observamos disminución de la profundidad del lecho, eritema e inflamación del tejido circundante, con delimitación de los bordes de la herida; así como blanqueamiento de la piel. Como se trataba de una herida crónica e infectada, la curación completa se produjo en sesenta y tres días. Se concluye que la combinación de acción fotodinámica con presión negativa estimulando la vascularización y oxigenación, además de mejorar las condiciones corporales con SVPBM, promovió una recuperación mucho más rápida que cada acción individual.

Palabras clave: Fotobiomodulación; Dehiscencia quirúrgica; Terapia fotodinámica.
\end{abstract}

\title{
1. Introdução
}

O emprego da Laserterapia de Baixa Potência permite diferentes abordagens clínicas. Utilizando doses baixas e moderadas, é possível irradiar, localmente, lesões e áreas com baixa oxigenação e acúmulo de toxinas. Aplicando altas doses, é possível irradiar sistemicamente o paciente, melhorando suas respostas fisiológicas em todo o organismo e, auxiliando, indiretamente, o tratamento das áreas traumatizadas ou com lesões, e, finalmente, também com doses mais altas, o emprego desses lasers, ainda que apresentem baixas potências (até $100 \mathrm{~mW}$ ), torna-se possível promover uma fotoativação de drogas fotossensíveis, previamente incorporadas nos tecidos-alvo, resultando numa reação fotodinâmica, e em controle microbiológico (terapia fotodinâmica antimicrobiana) (Lizarelli., 2018).

A fotobiomodulação (FBM) consiste na irradiação de células com fonte de luz não ionizante num determinado comprimento de onda, o qual pode levar a ativação de componentes celulares e promover reações fotoquímicas e fotofísicas que alteram o metabolismo celular, resultando no alívio da dor ou inflamação, imunomodulação, cicatrização de feridas e regeneração de tecidos podendo modular reações fisiológicas localmente e sistemicamente, através de reações fotoquímicas (Anders et al., 2015).

O leito da lesão e as bordas também poderão ser irradiados no intuito acelerar a neoformação dos tecidos (Petz et al., 2020). A terapia fotodinâmica antimicrobiana (TFDa) é uma reação onde a luz associada a uma substância fotossensibilizante, irá produzir uma reação fotodinâmica, por meio da produção de oxigênio altamente reativo, induzindo microrganismos a morte. O fotossensibilizador reage com as moléculas de oxigênio da célula, levando à produção de radicais livres, ou transferindo energia ou oxigênio, levando à produção de oxigênio singleto, causando distúrbio na parede celular e danos ao DNA, induzindo a morte celular e destruição do tecido comprometido (Eduardo et al., 2015; Aspiroz et al., 2017). Possui uma ação antimicrobiana e representa uma alternativa de tratamento para patógenos resistentes aos medicamentos convencionais, como alternativa para o tratamento de infecções multirresistentes e até o momento, não há relatos de microrganismos se tornando resistentes à TFDa (Giuliani et al., 2010; Maisch., 2009). Tem sido uma terapia importante no tratamento de feridas (Nesi-Reis et al., 2018; Petz et al., 2020).

O Vacum Laser (VL) é um aparelho que combina terapia a vácuo com FBM por meio de sistema de laser duplo (laser vermelho e infravermelho) em uma única plataforma, permitindo utilizar as duas terapias associadas ou separadamente, associando os benefícios da FBM ao efeito potencializador da vacuoterapia (Lopes et al., 2019) favorecendo diversos efeitos terapêuticos, como anti-inflamatório, analgésico e drenagem linfática (Rozenfeld \& Kalichman, 2016).

"Intravascular Laser Irradiation of Blood" (ILIB), foi uma terapia desenvolvida pelos cientistas soviéticos E. N. Meshalkina e V. Sergievskiy em 1981, para o tratamento de doenças cardiovasculares com evidências de suas propriedades reológicas e microcirculatórias. A irradiação com laser vermelho ou infravermelho diretamente nas células sanguíneas tem 
mostrado efeitos na melhora do sangue, da circulação linfática e da resposta imunológica quando a intenção é melhorar a resposta imunológica do paciente, a irradiação deve ser feita nos linfonodos que drenam a região lesionada (SemyachkinaGlushkovskaya et al., 2020; Arany et al., 2020). Também apresenta efeitos anti-inflamatórios, vasodilatação, melhora da deformabilidade eritrocitária, resultando em melhor suprimento de oxigênio, diminuindo o dióxido de carbono; melhora da hipóxia e normalização do tecido com aumento da síntese de ATP; efeito na liberação de óxido nítrico de monócitos. Tem mostrado reduzir o conteúdo de proteína $\mathrm{C}$ reativa, aumenta a atividade do complemento e o nível de imunoglobulina plasmática (IgA, IgM, IgG), além de diminuir a capacidade de agregação de trombócitos e ativa a fibrinólise que aumenta a circulação periférica (Weber et al., 2007; Kazemikoo et al., 2013; Tomimura et al., 2014; Deryugina et al., 2019). Hoje, o que promovemos são as abordagens transcutânea e transmucosa (Lizarelli et al., 2021), ou seja, não introduzimos um cateter dentro do vaso sanguíneo, porém entregamos a mesma energia necessária, corrigindo a potência e a irradiância entregue na pele, onde o vaso calibroso de interesse se aproxima mais da superfície. Para tanto, a terapia ganhou o nome de Fotobiomodulação Sistêmica Vascular Transcutânea (FSVTC).

As feridas cirúrgicas (FC) são classificadas como agudas por serem intencionais e planejadas; seu fechamento pode ser por primeira, segunda ou terceira intenção. A deiscência de FC (DFC) é uma complicação em que as margens da incisão são separadas, com ou sem exposição dos tecidos subjacentes, e pode se tornar complexa e crônica com complicações na cicatrização. A deiscência de FC impacta nas taxas de mortalidade e morbidade e contribui significativamente para estadias prolongadas em hospitais e estressores psicossociais a pacientes e familiares (Marques et al., 2016).

A cicatrização de feridas e a reparação de tecidos são processos complexos que envolvem uma série dinâmica de eventos, incluindo coagulação, inflamação, formação de tecido de granulação, epitelização, síntese de colágeno e remodelação de tecidos. A FBM tem sido eficaz em acelerar a cicatrização do tecido lesado, induzindo a proliferação celular e aumentando a síntese de ATP, ácido nucléico e colágeno (Anders et al., 2015).

Diversas associações tópicas podem ser utilizadas com a FBM, VL terapia e FSVTC, entre elas podemos citar o gel de Polihexametileno Biguanida (PHMB) muito utilizado para manter o meio úmido, além de realizar controle antimicrobiano, destruindo bactérias, vírus e fungos, com baixa toxicidade de menor risco de desenvolver resistênca (Alves et al., 2018).

O objetivo deste relato de caso é demonstrar que a associação da FBM, VL Terapia, TFDa, FSVTC e curativos tópicos no tratamento de deiscências de artroplastia de joelho, complementam-se em um processo de cicatrização.

\section{Metodologia}

Este relato de caso foi realizado de forma descritiva, com coleta de dados do tratamento da paciente por meio do prontuário eletrônico. Foram analisados os sinais e sintomas, apresentados pela paciente, bem como as características da ferida. Esse tipo de pesquisa é considerado uma fonte de informação importante e que pode fornecer subsídios para diferenciar o tratamento dos pacientes (Yoshida, 2007).

Este estudo foi aprovado pelo Comitê de Ética em Pesquisa da Faculdade de Medicina de Botucatu, Universidade Estadual Paulista (UNESP), Brasil (CAAE: 74104821.8.0000.5411). O consentimento informado foi assinado pelo paciente antes do início do estudo.

\section{Relato de Caso}

Jovem, 23 anos, sem comorbidades, foi submetida a artroplastia de joelho por ruptura do ligamento cruzado anterior por queda, em 03/12/2018. Após 10 dias houve deiscência da ferida operatória, sendo ressuturada. Em 23/12/2018 os pontos foram retirados com a finalidade de cicatrização por segunda intenção e indicado curativo tópico com gel de PHMB por 15 
dias sem melhora; sendo substituída por placa de alginato de cálcio e hidrofibra apresentando pouca melhora da DFC. Em 25/03/2019 evoluiu com pequenas áreas avermelhadas ao redor da lesão com diagnóstico de dermatite atópica relacionada ao estresse. Apresentou drenagem excessiva de exsudato em 20/04/2019, com suspeita de fístula de drenagem sinovial. Foi indicada a tala gessada do membro afetado, mantendo apenas uma abertura na DFC.

Em 27/04/2019 a paciente foi encaminhada ao ambulatório de laserterapia. Iniciou-se o tratamento com associação de curativos tópicos e com diferentes fontes de luz não ionizante (Figura 1). O protocolo está descrito na Tabela 1.

Figura 1. Aspecto inicial da deiscência da ferida cirúrgica com inflamação.

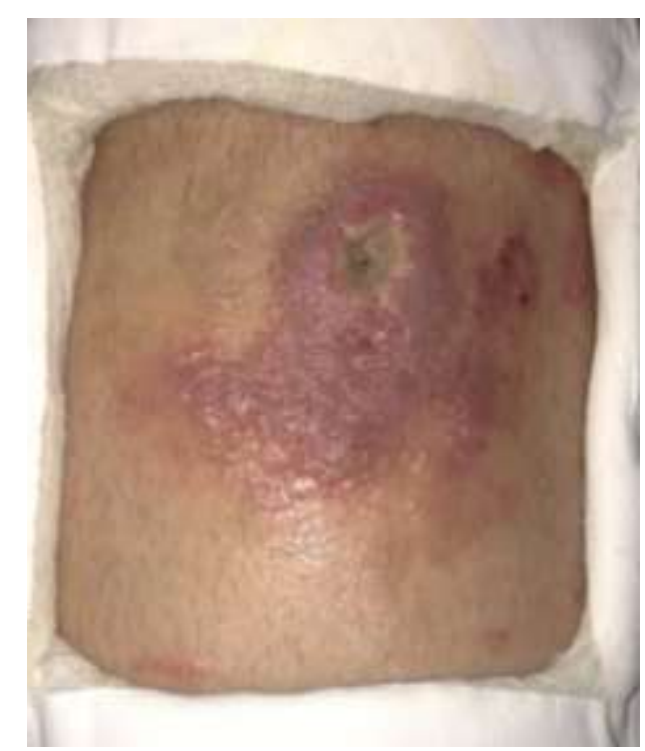

Fonte: Autores (2021).

A Figura 1 mostra a lesão em sua fase inicial do tratamento, quando foi encaminhado ao ambulatório de laserterapia. Iniciamos com o controle microbiológico da DFC com 3 sessões do VL terapia com TFDa no primeiro e quarto dias; e décimo terceiro dias para prevenir reinfecção. O fotossensibilizante utilizado foi a solução aquosa de azul de metileno diluído em água destilada por osmose reversa 0,01\% (Pharma Blue 10, PDT Pharma, Cravinhos, SP, Brasil), o qual foi aplicado no leito da ferida com tempo de pré-irradiação de 10 minutos.

À seguir, foi aplicada a vacuoterapia associada a fotobiomodulação (Vacumlaser, MMO, São Carlos, SP, Brasil) por 30 segundos, resultando numa energia total de $18 \mathrm{~J}$, quando apenas os três diodos laser emitindo no vermelho $(660 \mathrm{~nm}), 100 \mathrm{~mW}$ de potência cada, ficavam ligados. A ventosa acrílica utilizada tinha $40 \mathrm{~mm}$ de diâmetro e a pressão negativa empregada foi de 50mbar (Figura 2). O objetivo inicial foi de fotoativar a solução de azul de metileno, resultando na indução da morte dos microrganismos através da terapia fotodinâmica antimicrobiana.

A Figura 2 demonstra a aplicação da solução aquosa de azul de metileno a $0,01 \%$ e a sua fotoativação empregando a vacuolaserterapia por 30 segundos. 
Figura 2. Aplicação da terapia fotodinâmica: deposição da solução aquosa de azul de metileno (a) fotoativada pela vacuolaserterapia (b).
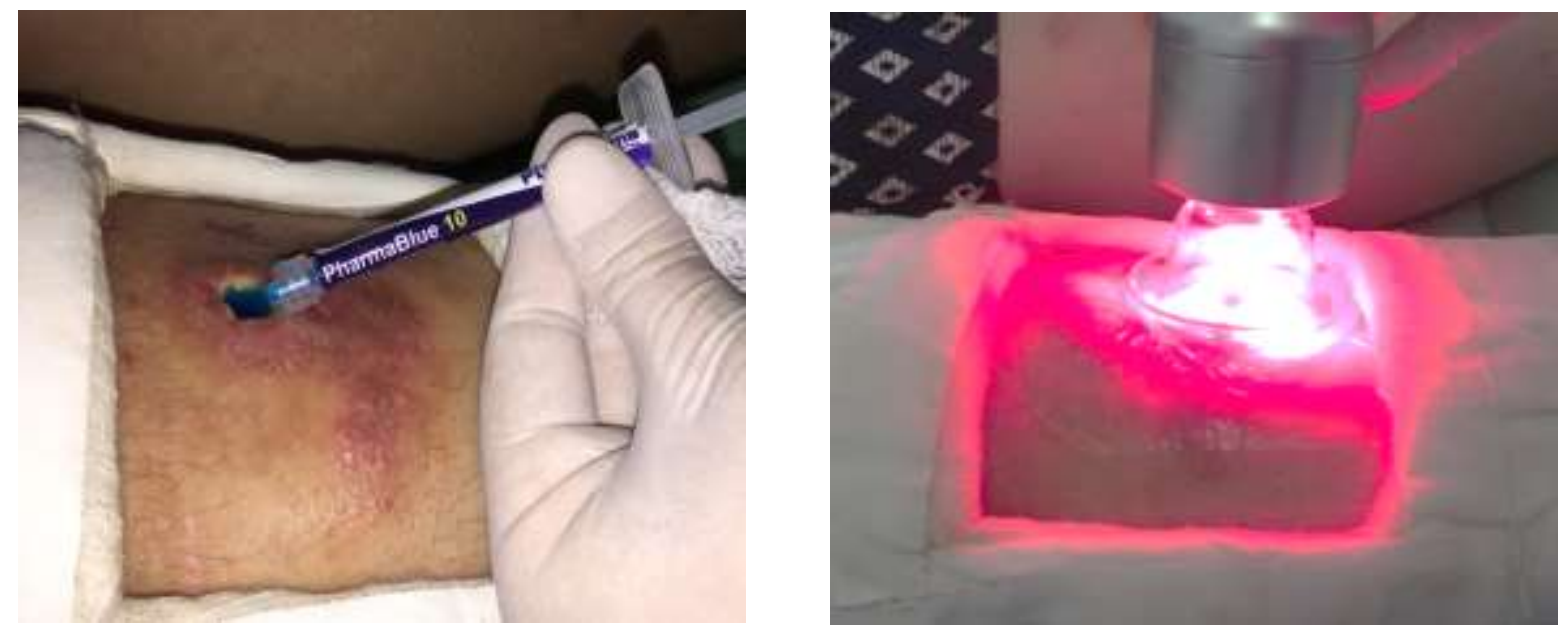

Fonte: Autores (2021).

A segunda fase foi a FBM local e sistêmica em que utilizamos Laser Duo (100mW, área spot 3mm², comprimentos de onda 660nm e 808nm, MMOptics, São Carlos, SP, Brasil). Para FBM local aplicamos por contato pontos equidistantes de 2 $\mathrm{cm}, 660 \mathrm{~nm}$ no leito da ferida e $808 \mathrm{~nm}$ ao redor da lesão com doses de $2 \mathrm{~J}$ e $3 \mathrm{~J}$ por ponto para o laser vermelho e infravermelho, respectivamente. O número de pontos foram diminuindo conforme a ferida cicatrizava. Após as sessões foram realizados curativos primários com PHMB gel (30 ml, CURATEC@)).

Após 8 dias observamos uma diminuição da profundidade do leito, do eritema e da inflamação do tecido ao redor com delimitação das bordas da ferida; bem como o clareamento da pele (Figura 3).

Figura 3. Diminuição da profundidade do leito, eritema e inflamação, delimitação das bordas e clareamento da pele.

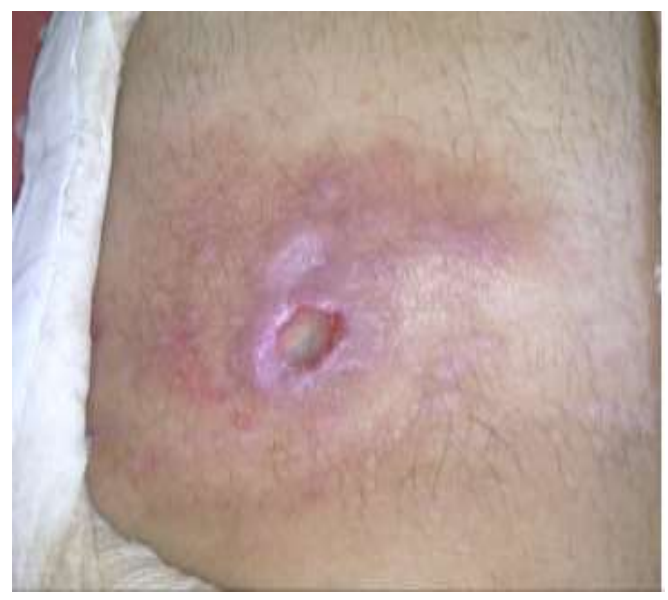

Fonte: Autores (2021).

A FSVTC foi realizada duas vezes por semana, alternando os comprimentos de onda vermelho (660nm) e infravermelho $(808 \mathrm{~nm})$. As quatro primeiras sessões foram realizadas na artéria radial do braço esquerdo por 60 minutos, resultando em 360J (Figura 4). Após a remoção do gesso as aplicações foram feitas alternando, a cada sessão, as artérias poplítea e tibial anterior direita (30 minutos, 180J), com a finalidade de irradiar grandes vasos próximos ao local da ferida.

Na figura 5, observamos a evolução da cicatrização da ferida com laser vermelho e infravermelho, aplicados localmente, no leito da ferida e nas bordas e associando a FSVTC nas artérias poplítea e tibial anterior. A cicatrização seguiu 
suas etapas de fechamento por segunda intenção e remodelação tecidual com rapidez e sem gerar deformidade na região afetada.

Figura 4. Fotobiomodulação sistêmica vascular transcutânea por 60 minutos na artéria radial.

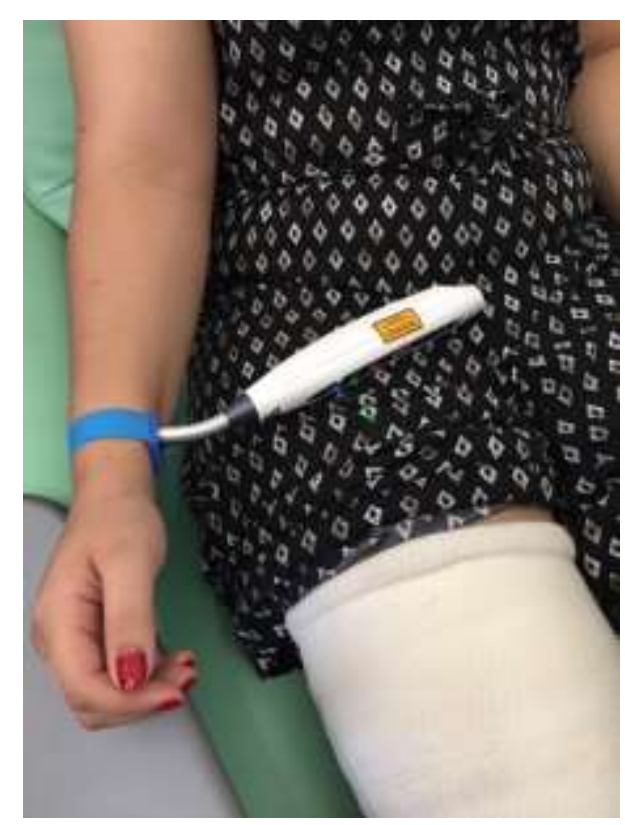

Fonte: Autores (2021).

Figura 5. Evolução da ferida sob terapia com laser vermelho e infravermelho (FBM): 21 dias (17/05/2019) (A), 34 dias (31/05/2019) (B), 40 dias (08/06/2019) (C), e, 60 dias (28/06/2019) (D).

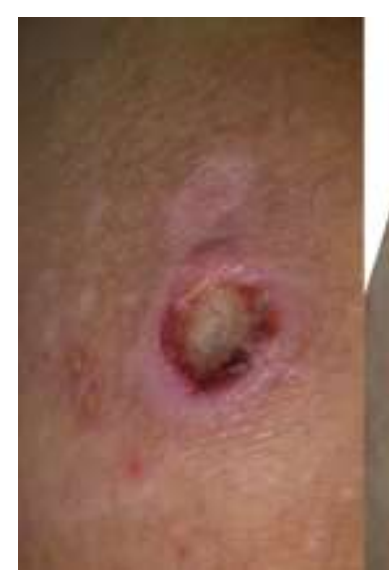

$\mathrm{a}$

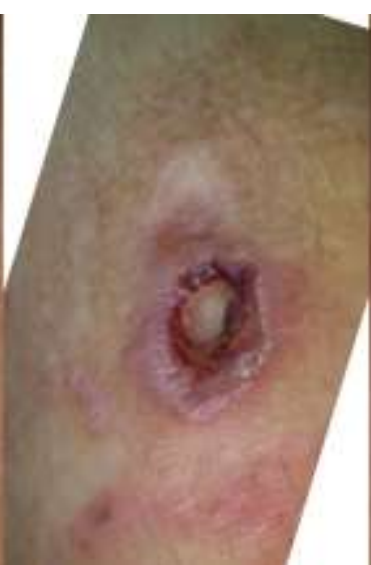

b

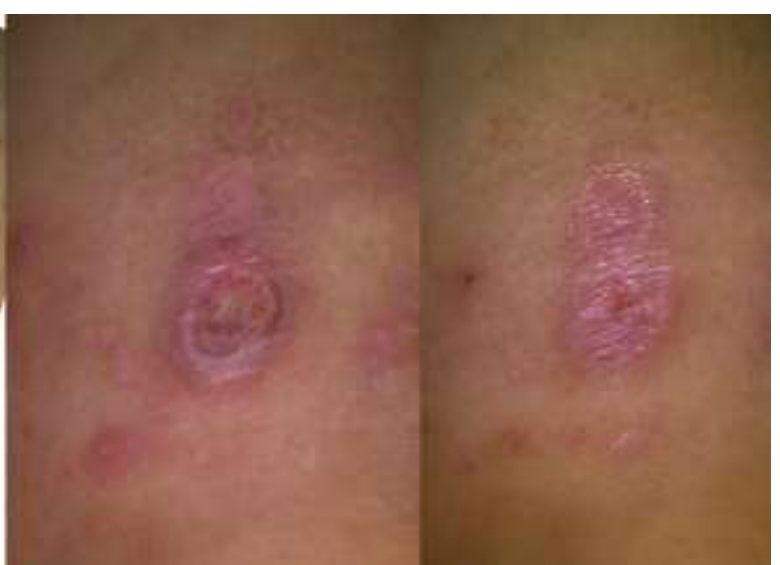

c

d

Fonte: Autores (2021). 
Tabela 1 - Protocolo de tratamento das deiscências de ferida cirúrgica decorrente da artroplastia de joelho.

\begin{tabular}{|c|c|c|c|c|}
\hline Data & FBM & VL com PDT & FSVTC & Curativo tópico \\
\hline 27/04/2019 & & $18 \mathrm{~J}, 660 \mathrm{~nm}$ & $\begin{array}{l}660 \mathrm{~nm}, 60 \text { minutos } \\
\text { artéria radial } \mathrm{E}\end{array}$ & \\
\hline $30 / 04 / 2019$ & & $18 \mathrm{~J}, 660 \mathrm{~nm}$ & $\begin{array}{l}808 \mathrm{~nm}, 60 \text { minutos } \\
\text { artéria radial } \mathrm{E}\end{array}$ & PHMB gel \\
\hline 04/05/2019 & $\begin{array}{l}660 \mathrm{~nm} 2 \mathrm{~J} \text { leito ferida, } 808 \mathrm{~nm} \\
3 \mathrm{~J} \text { ao redor ferida diariamente }\end{array}$ & & $\begin{array}{l}808 \mathrm{~nm} / 60 \text { minutos } 2 \mathrm{x} \\
\text { semana - artéria radial } \mathrm{E}\end{array}$ & PHMB gel \\
\hline $10 / 05 / 2019$ & $\begin{array}{l}660 \mathrm{~nm} 2 \mathrm{~J} \text { leito ferida, } 808 \mathrm{~nm} \\
3 \mathrm{~J} \text { ao redor ferida diariamente }\end{array}$ & & $\begin{array}{l}808 \mathrm{~nm} / 60 \text { minutos } 2 \mathrm{x} \\
\text { semana - artéria radial E }\end{array}$ & PHMB gel \\
\hline $17 / 05 / 2019$ & & $18 \mathrm{~J}, 660 \mathrm{~nm}$ & $\begin{array}{l}30 \text { minutos, diários, } \\
\text { alternando } 660 \mathrm{~nm} \text { e } 808 \mathrm{~nm} \text {, } \\
\text { arteriais poplítea e tibial } \\
\text { anterior direita }\end{array}$ & PHMB gel \\
\hline $31 / 05 / 2019$ & $\begin{array}{l}660 \mathrm{~nm} 2 \mathrm{~J} \text { leito ferida, } 808 \mathrm{~nm} \\
3 \mathrm{~J} \text { ao redor ferida diariamente }\end{array}$ & & & PHMB gel \\
\hline 08/06/2019 & $\begin{array}{l}660 \mathrm{~nm} 2 \mathrm{~J} \text { leito ferida, } 808 \mathrm{~nm} \\
3 \mathrm{~J} \text { ao redor ferida diariamente }\end{array}$ & & $\begin{array}{l}30 \text { minutos, diários, } \\
\text { alternando } 660 \mathrm{~nm} \text { e } 808 \mathrm{~nm} \text {, } \\
\text { artérias poplítea e tibial } \\
\text { anterior direita }\end{array}$ & PHMB gel \\
\hline $15 / 06 / 2019$ & $\begin{array}{l}660 \mathrm{~nm} 2 \mathrm{~J} \text { leito ferida, } 808 \mathrm{~nm} \\
3 \mathrm{~J} \text { ao redor ferida diariamente }\end{array}$ & & $\begin{array}{l}\text { 660nm / } 30 \text { min diários em } \\
\text { cada artéria poplítea e tibial } \\
\text { anterior direita }\end{array}$ & PHMB gel \\
\hline 23/06/2019 & $\begin{array}{l}660 \mathrm{~nm} 2 \mathrm{~J} \text { leito ferida, } 808 \mathrm{~nm} \\
3 \mathrm{~J} \text { ao redor ferida diariamente }\end{array}$ & & $\begin{array}{l}660 \mathrm{~nm} / 30 \text { min diários em } \\
\text { cada artéria poplítea e tibial } \\
\text { anterior direita }\end{array}$ & PHMB gel \\
\hline 28/06/2019 & $\begin{array}{l}660 \mathrm{~nm} 2 \mathrm{~J} \text { leito ferida, } 808 \mathrm{~nm} \\
3 \mathrm{~J} \text { ao redor ferida diariamente }\end{array}$ & & $\begin{array}{l}\text { 660nm /30 min diários em } \\
\text { cada artéria poplítea e tibial } \\
\text { anterior direita }\end{array}$ & PHMB gel \\
\hline
\end{tabular}

Fonte: Autores (2021).

\section{Discussão}

A FBM tem se mostrado eficiente no tratamento de diversas lesões. Estudos evidenciam resultados importantes no processo da cicatrização de feridas em pacientes diabéticos, queimaduras, epidermólise bolhosa, entre outras (Carvalho et al., 2016; Kazemikazemikoo et al., 2018; Minicucci et al., 2010).

Pesquisas in vitro e in vivo reportam não apenas os mecanismos moleculares pelos quais a FBM forma espécies reativas de oxigênio, ativa enzimas, principalmente ATPases; estimula o cálcio e a mitose e aumenta a formação de mRNA e secreção de proteína, mas também o mecanismo pelo qual estimula a produção de colágeno, aumenta a proliferação e motilidade fibroblástica e de queratinócitos, proliferação capilar e epitelização (Schindl et al., 1999; Grosmman et al., 1998; Schindl et al., 2002).

A lesão inicial deste relato de caso, apresentava com quadro de inflamação crônica, estase de toxinas e dificuldade de cicatrização, sendo necessárias três condutas: controle microbiológico, drenagem linfática e aumento da oxigenação. Sugerimos que a melhora significativa da ferida após uma semana tenha sido decorrente da VL terapia, que possibilitou uma rápida drenagem das toxinas e melhora da circulação sanguínea e linfática, por meio da pressão negativa, além do aumento da atividade mitótica caracterizado pela melhora do leito da FC. A associação com a TFDa foi para permitir melhores condições de cicatrização tecidual, por meio da descontaminação inicial, bem como evitar processos de reinfecções. Oliveira et al (2020) citam a FBM e a vacuoterapia como avanços terapêuticos para feridas. A utilização de duas terapias associadas ou separadas, aumentam os benefícios da FBM bem como potencializam os efeitos da terapia a vácuo (Lopes et al., 2019). 
No tecido infectado pode ocorrer um déficit na circulação sanguínea causando a hipóxia celular, fazendo com que as células não recebessem nutrientes suficientes. Nesse caso clínico, a vacuoterapia, por meio da combinação da pressão arterial positiva com a pressão negativa do vácuo, potencializou a FBM em diversas condições clínicas, intensificando a qualidade e a velocidade da resposta biológica do tecido ao tratamento.

A associação da FBM local e sistêmica, foi importante para a modulação da sinalização redox na cadeia respiratória, fato esse que corrobora o que os autores Huang et al. (2012); Salehpour et al. (2019) referem em seus estudos, que pode haver indução de efeitos positivos na expressão de interleucinas, interferons e imunoglobulinas, além de aumentar a diferença arteriovenosa de oxigênio. Tal fato pode ser capaz de acabar com a hipóxia tecidual e promover o enriquecimento de oxigênio, que é um sinal de normalização do metabolismo tecidual, aumentando a síntese de ATP com uma normalização do potencial de membrana celular, fatores esses fundamentais para a cicatrização (Huang et al., 2012; Salehpour et al., 2019).

A FBM é constantemente utilizada em deiscência de ferida cirúrgica, principalmente em pacientes que foram submetidos a esternotomia longitudinal. Em estudo realizado por Dixit et al. (2013), o uso da FBM foi considerado como um novo método para tratamento da deiscência, melhorando a qualidade de vida do paciente no pós operatório.

Para que uma terapia tenha os resultados esperados, é necessário que várias situações limitantes sejam superadas. No caso da recuperação da cicatrização de feridas, limitações como infecção, presença de tecido inviável, falta de circulação e situação desfavorável do paciente como um todo, são algumas das limitações. Com base nessas limitações, o sucesso na cura requer uma combinação de técnicas. Um deles, sozinho, ajuda a resolver o problema, porém necessita do sinergismo da outra terapia para ser mais eficaz. O emprego da FBM é excelente, mas pode ser muito melhor se houver oxigenação dos tecidos, ausência de infecção e condições corporais adequadas (Lizarelli, 2018).

\section{Conclusão}

Conclui-se que que a combinação da ação fotodinâmica com pressão negativa estimulando a vascularização e a oxigenação bem como a melhora das condições corporais com SVPBM, promoveu uma recuperação muito mais rápida do que cada ação individual.

Esse caso pode ser um estímulo para a implementação de protocolos mais elaborados que tragam ao paciente uma recuperação muito melhor.

\section{Referências}

Alves, I. L. M., Santana, L. A., Neves, R. S., Guadagnin, R. V. \& Araújo, G. S. A. (2018). A efetividade da Polihexanida (PHMB) na cicatrização de lesões por pressão: um estudo preliminar. Revista Feridas, 06(30), 1003-1007. http://www.revistaferidas.com.br/revistas/ed30/Revista_Ferid as_30_Completa.pdf\#page $=36$

Anders, J. J., Lanzafame, R. J., \& Arany, P. R. (2015). Low-level light/laser therapy versus photobiomodulation therapy. Photomedicine and laser surgery, 33(4), 183-184. https://doi.org/10.1089/pho.2015.9848

Arany P. R. (2020). Photoimmunotherapy: A Novel Field with Overlapping Light Treatment Approaches. Photobiomodulation, photomedicine, and laser surgery, 10.1089/photob.2020.4877. Advance online publication. https://doi.org/10.1089/photob.2020.4877

Aspiroz, C., Sevil, M., Toyas, C. \& Gilaberte, Y. (2017). Terapia fotodinâmica con azul de metileno en úlceras cutáneas infectadas con Psedomonas aeruginosas y Fusarium spp. Actas Dermosifiliogr, 108(6), 45-48. https://www.sciencedirect.com/science/article/abs/pii/S0001731017300777

Carvalho, A. F., Feitosa, M. C., Coelho, N. P., Rebêlo, V. C., Castro, J. G., Sousa, P. R., Feitosa, V. C., \& Arisawa, E. A. (2016). Low-level laser therapy and Calendula officinalis in repairing diabetic foot ulcers. Revista da Escola de Enfermagem da U S P, 50(4), 628-634. https://doi.org/10.1590/S0080623420160000500013

Deryugina, A. V., Ivashchenko, M. N. \& Ignatiev, P. S. Irina V. Balalaeva, I.V. \& Samodelkin, A. G. (2019). Low-level laser therapy as a moifier of erythrocytes morphokinetic parameters in hyperadrenalinemia. Lasers in Medical Scienc, e34, 1603-1612. https://doi.org/10.1007/s10103-019-02755-y

Dixit, S., Maiya, A., Umakanth, S., \& Borkar, S. (2013). Fotobiomodulação da deiscência de ferida operatória em diabético por laserterapia de baixa intensidade após esternotomia mediana. Jornal indiano de cuidados paliativos, 19 (1), 71-75. https://doi.org/10.4103/0973-1075.110242 
Eduardo, A. Bello-Silva, M. S., Ramalho, K. M., Lee, E. M. R. \& Aranha, A. C. C. (2015). Terapia fotodinâmica como benefício complementar na clínica odontológica Rev Assoc Paul Cir Dent, 69(3), 226-35. http://revodonto.bvsalud.org/pdf/apcd/v69n3/a04v69n3.pdf

Giuliani, F., Martinelli, M., Cocchi, A., Arbia, D., Fantetti, L., \& Roncucci, G. (2010). In vitro resistance selection studies of RLP068/Cl, a new Zn(II) phthalocyanine suitable for antimicrobial photodynamic therapy. Antimicrobial agents and chemotherapy, 54(2), 637-642. https://doi.org/10.1128/AAC.00603-09

Grossman, N., Schneid, N., Reuveni, H., Halevy, S., \& Lubart, R. (1998). $780 \mathrm{~nm}$ low power diode laser irradiation stimulates proliferation of keratinocyte cultures: involvement of reactive oxygen species. Lasers in surgery and medicine, 22(4), 212-218. https://doi.org/10.1002/(sici)10969101(1998)22:4<212::aid-lsm5>3.0.co,2-s

Huang, S. F., Tsai, Y. A., Wu, S. B., Wei, Y. H., Tsai, P. Y., \& Chuang, T. Y. (2012). Effects of intravascular laser irradiation of blood in mitochondria dysfunction and oxidative stress in adults with chronic spinal cord injury. Photomedicine and laser surgery, 30(10), 579-586. https://doi.org/10.1089/pho.2012.3228

KazemiKhoo, N., Iravani, A., Arjmand, M., Vahabi, F., Lajevardi, M., Akrami, S. M., \& Zamani, Z. (2013). A metabolomic study on the effect of intravascular laser blood irradiation on type 2 diabetic patients. Lasers in medical science, 28(6), 1527-1532. https://doi.org/10.1007/s10103-012-1247-4

Kazemikhoo, N., Vaghardoost, R., Dahmardehei, M., Mokmeli, S., Momeni, M., Nilforoushzadeh, M. A., Ansari, F., Razagi, M. R., Razagi, Z., Amirkhani, M. A., \& Masjedi, M. R. (2018). Evaluation of the Effects of Low-Level Laser Therapy on the Healing Process After Skin Graft Surgery in Burned Patients (A Randomized Clinical Trial). Journal of lasers in medical sciences, 9(2), 139-143. https://doi.org/10.15171/jlms.2018.26

Lizarelli, R. F. Z. (2018). Reabilitação biofotônica orofacial: fundamentos e protocolos clínicos. Compacta

Lizarelli, R. F. Z., Grecco, C., Regalo, S. C. H., Esteban Florez, F. L., \& Bagnato, V. S. A pilot study on the effects of transcutaneous and transmucosal laser irradiation on blood pressure, glucose and cholesterol in women. Heliyon. 7(5):e07110. https://10.1016/j.heliyon.2021.e07110

Lopes, L. A. B., Alvarez, C. \& Paolillo, F. R. Protocolos clínicos em reabilitação. (2a ed.) https://mmo.com.br/protocolos/!\#4-19-vacum-laser-1578711777

Maisch T. (2009). A new strategy to destroy antibiotic resistant microorganisms: antimicrobial photodynamic treatment. Mini reviews in medicinal chemistry, 9(8), 974-983. https://doi.org/10.2174/138955709788681582

Marques, G., Almeida, P., de Farias, L., \& do Nascimento, D. (2017). Estudo preliminar sobre registros de deiscência de ferida operatória em um hospital universitário. Revista Hospital Universitário Pedro Ernesto 15(4), 312-319. doi:https://doi.org/10.12957/rhupe.2016.31605

Minicucci, E. M., Barraviera, S. R., Miot, H., Almeida-Lopes, L. (2010). Low-level laser therapy for the treatment of epidermolysis bullosa: case report. J Cosmet Laser Ther, 12, 203-205. https://doi.org/10.3109/14764172.2010.502460

Nesi-Reis, V., Lera-Nonose, D., Oyama, J., Silva-Lalucci, M., Demarchi, I. G., Aristides, S., Teixeira, J., Silveira, T., \& Lonardoni, M. (2018). Contribution of photodynamic therapy in wound healing: A systematic review. Photodiagnosis and photodynamic therapy, 21, 294-305. https://doi.org/10.1016/j.pdpdt.2017.12.015

Oliveira, A., Simões, S., Ascenso, A., \& Reis, C. P. (2020). Therapeutic advances in wound healing. The Journal of dermatological treatment, 1-21. Advance online publication. https://doi.org/10.1080/09546634.2020.1730296

Petz, F., Félix, J., Roehrs, H., Pott, F. S., Stocco, J., Marcos, R. L., \& Meier, M. J. (2020). Effect of Photobiomodulation on Repairing Pressure Ulcers in Adult and Elderly Patients: A Systematic Review. Photochemistry and photobiology, 96(1), 191-199. https://doi.org/10.1111/php.13162

Rozenfeld, E., \& Kalichman, L. (2016). New is the well-forgotten old: The use of dry cupping in musculoskeletal medicine. Journal of bodywork and movement therapies, 20(1), 173-178. https://doi.org/10.1016/j.jbmt.2015.11.009

Salehpour, F., Gholipour-Khalili, S., Farajdokht, F., Kamari, F., Walski, T., Hamblin, M. R., DiDuro, J. O., \& Cassano, P. (2020). Therapeutic potential of intranasal photobiomodulation therapy for neurological and neuropsychiatric disorders: a narrative review. Reviews in the neurosciences, 31(3), 269-286. https://doi.org/10.1515/revneuro-2019-0063

Schindl, A., Heinze, G., Schindl, M., Pernerstorfer-Schön, H., \& Schindl, L. (2002). Systemic effects of low-intensity laser irradiation on skin microcirculation in patients with diabetic microangiopathy. Microvascular research, 64(2), 240-246. https://doi.org/10.1006/mvre.2002.2429

Schindl, A., Schindl, M., Pernerstorfer-Schon, H., Kerschan, K., Knobler, R. \& Schindl, L. (1999). Diabetic neuropathic foot ulcer: successful treatment by low-intensity laser therapy. Dermatology, 198:314-316. https://doi.org/10.1159/000018140

Semyachkina-Glushkovskaya, O., Abdurashitov, A., Dubrovsky, A., Klimova, M., Agranovich, I., Terskov, A., Shirokov, A., Vinnik, V., Kuzmina, A., Lezhnev, N., Blokhina, I., Shnitenkova, A., Tuchin, V., Rafailov, E., \& Kurths, J. (2020). Photobiomodulation of lymphatic drainage and clearance: perspective strategy for augmentation of meningeal lymphatic functions. Biomedical optics express, 11(2), 725-734. https://doi.org/10.1364/BOE.383390.

Tomimura, S. Silva, B. P. A., Sanches, I. C., Canal, M, Consolim-Colombo, F., Conti, F. F, De Angelis, K., Chavantes, M. C., (2014). Efeito hemodinâmico da laserterapia em ratos espontaneamente hipertensos. Arq Bras Cardiol, 103(2), 161-164. https://www.scielo.br/j/abc/a/BR5SQYpCxvvRKM 7B7pMJzYk/abstract/?lang=pt

Weber, M. H. (2006). The intravenous laser blood irradiation - introduction of a new therapy. 657-699, Chapter I. https://www.isla-laser.org/wpcontent/uploads/Chapter-Weber-final.pdf.

Yoshida, W. B. (2007). Redação do relato de caso. J Vasc Bras. 6(2). https://doi.org/10.1590/S1677-54492007000200004 